\title{
Surveillance of antibiotic use and resistance in orthopaedic department in an Egyptian university hospital
}

\author{
Azza M. Hassan' ${ }^{1}$ Omaima Ibrahim², Magdy El Guinaidy ${ }^{3}$ \\ ${ }^{1}$ Microbiology and Immunology Department, Faculty of Medicine, Tanta University, Egypt \\ ${ }^{2}$ Public Health Department, Faculty of Medicine, Al-Azhar University, Egypt \\ ${ }^{3}$ Internal Medicine Department, Faculty of Medicine, Ain Shams University, Eygpt and the President of the Arab \\ Society of Infectious Diseases and Antimicrobials (ASIDA)
}

doi: 10.3396/ijic.V7i1.006.11

\begin{abstract}
Antimicrobial use and subsequently antimicrobial resistance among bacterial pathogens is a global problem, but in Egypt data are sparse. Orthopaedic departments reflect this problem because in them surgery constitutes the main manipulation and septic diseases constitute common complications both need guided antimicrobial therapy otherwise antimicrobial resistance will emerge. In order to highlight these issues, a prospective 9-month study on antimicrobial use and the antimicrobial susceptibility patterns of the isolated organisms in Orthopaedic Department, Tanta University, Egypt was carried out. High antimicrobial prescription rates $(98.1 \%)$ with low rates of appropriateness $(11.3 \%)$ were detected. Eighty-one percent of prescribed antimicrobial agents belong to cephalosporins and penicillins classes. Antibiotics were given for prophylaxis continued post-operatively $(77 \%)$, for infections $(13.4 \%)$ as well as randomly $(22 \%)$. High rates of resistance were found in most of the bacteria studied. 53.3\% of Staphylococcus aureus (S. aureus) and $66.7 \%$ of coagulase-negative Staphylococcus (CNS) isolates were oxacillin resistant. Multi-resistant strains represented $48.6 \%$ of the isolated gram negative strains of which $29.2 \%$ were ESBL (Klebsiella $62.5 \%$ and E. coli 33.3\%) while 19.4\% were MR Pseudomonas and Acinetobacter isolates. Antibiotic treatment in our Orthopaedic Department appears to be substantial. Increasing and justifying efforts are needed to improve appropriateness of antimicrobial therapy and minimize the development of antimicrobial resistance. We also call for a nationwide surveillance programme to monitor microbial trends and antimicrobial resistance patterns in Egypt.
\end{abstract}

Key words

Antibiotic use, ESBL, multidrug resistance, MRSA, Orthopaedic

Corresponding author

Azza M. Hassan

Assistant Professor of Microbiology and Immunology, Microbiology

and Immunology Department, Faculty of Medicine, Tanta University, Egypt.

Tel: 0020402241772 Mobile: 0020101052017 E-mail: azza1_9@hotmail.com 


\section{Introduction}

In spite of the availability of newer antibiotics, emerging antimicrobial resistance has become an increasing problem in the treatment of many hospital pathogens throughout the world, contributing to increased morbidity, patient length-of-stay, and, ultimately, mortality rates. ${ }^{1}$ Widespread use of antimicrobial agents has been accused to be a cause of emergence of such bacterial resistance. ${ }^{2}$ Moreover, researchers argue that the emergence of resistance usually reflects local practices. Therefore, surveys of antimicrobial use rates and the prevalence of drug resistance among bacteria in hospitals are needed to provide data for specific departments, rather than to adjust for the case mix in the whole hospital. ${ }^{1,3}$

Unfortunately, data regarding endemic antimicrobial resistance in Egypt, where over-the-counter antibiotic use is common, are unavailable. ${ }^{4}$ In orthopaedic surgery and trauma these problems are obvious as beside surgery which constitutes the main manipulation and the usual healthcare associated infections that commonly occur in surgical wards, specific septic diseases that usually requires high antibiotic doses and long term treatment represent the worst complications because their outcome can be devastating, resulting in total loss of joint function, re-operative intervention, amputation and, occasionally, death. ${ }^{5}$

Knowledge of used antibiotics and local antimicrobial resistance patterns are essential to guide empirical and pathogen-specific therapy, critical for optimal decisions regarding infection control policies and help assessing the magnitude of the resistance problem. ${ }^{4,6}$ Thus, the present study was undertaken in our Orthopaedic Department to define the pattern and appropriateness of antibiotic use, identify the infecting micro-organisms and determine the resistance patterns among the isolated bacteria.

\section{Patients, materials and methods}

\section{Study design}

This study is a 9-months, prospective study conducted from the start of January, 2008 to the end of September, 2008 on antibiotic use in Orthopaedic Department, Tanta University Hospital, Egypt. The infection rate and incidence of antimicrobial resistance among the possible isolated organisms was detected during the same period. This department, collectively, admit patients from all socioeconomic strata from Tanta and the surrounding rural areas. It is a 83-bed department containing 5 wards and 2 operation theatres with 3 operation's tables. The department doses not follow any definite antimicrobial protocols.

All cases who were admitted to this department during the study period are enrolled including both sexes and all ages. Approval of the local research ethics committee was obtained. One of the investigators, the microbiologist, visited the department at least 4 times a week and reviewed the patients' files. On each visit, the number of admitted patients was recorded as well as the number of patients receiving antibiotics. Demographic and clinical data of those patients were extracted and entered into patient-specific sheet. Obtained data included the patient's name, age, sex, past history, date of admission, date of discharge, diagnosis, details of operation (if any), details of infection (type, site and whether or not the culture was done before starting antibiotic therapy) details of antibiotic used (name, dose, route, time, duration, reason of use and number of courses) and baseline serum creatinine. For accurate microbiological diagnosis, samples were collected from infected cases and were subjected to standard microbiological examination.

Infections developed 72 hours or more after admission were considered healthcare associated infections. The indication of use of antibiotics was classified into prophylactic, empirical and therapeutic as well as randomly where antibiotics were given without evidence or suspicion of infection. ${ }^{7}$ Antimicrobial use was evaluated according to indication of administration, source of infections, and appropriateness of treatment. Antimicrobial therapy was followed from initiation through possible adaptations, until discontinuation of treatment.

Microbiological examination: identification of infected cases and the study of the resistance pattern of bacterial isolates were done in Microbiology Department, Tanta faculty of medicine. The specimens were collected, stained and cultured and the bacterial isolates were identified using standard microbiological procedure. $^{8}$ The performance of antibiotic sensitivity 
test and the choice of disks for each isolated organism and the interpretation of zone diameters results were guided by CLSI guidelines. ${ }^{9}$ Quality control strains were routinely utilized to ensure accurate assays. The isolates showing intermediate resistance were few and were grouped together with sensitive isolates for the purpose of data analysis. Methicillin resistance was detected by oxacillin disks. The diagnosis and conformation of ESBL was preliminary according to new and updated protocols for AST, 2005. ${ }^{10}$

\section{Data analysis}

Data were collected and tabulated as numbers and percentages or as mean $\pm \mathrm{SD}$. Appropriateness of therapy used is evaluated according to $\mathrm{WHO}$ guidelines. ${ }^{11}$ The appropriateness of $\mathrm{ABP}$ is determined by type, time, way of administration and duration of antibiotic used. The appropriate ABP should belong to the first or second generation cephalosporins (cefazolin or cefuroxime) with addition of gram negative organism coverage in type-III fracture that administered intravenously within sixty minutes prior to the incision and for a maximum of 48-hours post-operatively except in patients with type-III fracture where 5-10 days post operative prophylaxis is recommended. On the other hand, the appropriateness of Empiric/therapeutic antibiotic therapy should be adjusted according to the source of infection (nosocomial or acquired), site of infection (SSI, UTI, RTI and/or BSI) age of the patient (adult or child) and underlying kidney diseases guided by serum creatinine level. Antibiotic resistance is based on the type of the bacteria, location of the infection and the concentration of the antibiotic within the body.

Prescription rate was calculated by dividing the number of antimicrobial agents prescribed in each category (prophylactic, empiric, etc) on the total number of antibiotics prescribed multiplied by 100 . The appropriateness of therapy used was calculated by dividing the number of appropriate antimicrobial agents prescribed on the total number of antibiotics prescribed multiplied by 100 . Antibiotic resistance rate was determined by dividing the number of resistant isolate on total number of such isolate for each antibiotic used.

\section{Results}

Out of 671 patients who were admitted to the Orthopaedic Department during the study period, 32 patients were excluded because their records were irretrievable. Of the 639 surveyed hospitalized patients, 627 were received antimicrobial therapy, with a percentage of $98.1 \%$. A total of 1119 antibiotics were given to 627 patients, of whom $258(41.1 \%)$ were female and $369(58.9 \%)$ male. Of the patients receiving antibiotics, $53.1 \%$ were $\geq 61$ years old, $24.4 \%$ were $21-60$ years and $22.5 \%$ were $\leq 20$ years. Of notice, the infection rate was $13.1 \%$ where nosocomial infections represent the majority of the cases with a percentage of $10.3 \%$, mainly in the form of SSI $(4.7 \%)$ (Table I).

Twenty-one antimicrobial agents were prescribed as single agent (486 prescriptions) and as drug combinations (633 prescriptions in 273 combinations; double: 186 or triple: 87 ). The main duration of treatment was $12.4 \pm 10.9$ with a range of $3-37$ days. The most frequently prescribed antimicrobial classes are shown in figure 1 and the most frequently prescribed antimicrobial agents are shown in table II. Eighty-one percent of prescribed antimicrobial agents belong to cephalosporins and penicillins classes and $54.2 \%$ of all antimicrobial use consisted of cefotaxime (42.1\%) and amoxicillin-flucloxacillin (12.1\%). Of the total of 1119 antimicrobial drug courses, 213 (19\%) were entirely provided orally, $243(21.7 \%)$ intramuscularly (I.M) and 663 (59.3\%) intravenously (I.V).

Antibiotics were given for prophylaxis continued postoperatively $(483 ; 77 \%)$, for infections $(84 ; 13.4 \%$ : community $18 ; 2.9 \%$ and hospital $66 ; 10.5 \%$ ) and randomly $(138 ; 22 \%)$. The antimicrobial prescription rate for these groups was $66.8 \%, 18.5 \%$ and $14.7 \%$ respectively. Of notice, there was an overlap in antibiotic courses as 12 out of 18 patients who were admitted with infections have received empirical/therapeutic then prophylactic antibiotic courses. Similar overlap occurred in the 66 nosocomially infected patients.

The overall appropriateness of treatment was $11.3 \%$, with a range of $4.8-71.9 \%$ per course. Of notice, 165 prescriptions were given randomly without any indication. All ABP were given by intravenous rout. The appropriateness of $\mathrm{ABP}(36 ; 4.8 \%)$ was based on its type $(45 ; 6 \%)$, duration $(69 ; 9.2 \%)$ and timing $(93 ; 12.4 \%)$ 


$\begin{array}{lll}\square \text { Cephalosporins } & \square \text { Pencillins } & \square \text { Quinolones } \\ \square \text { Aminoglycosides } & \square \text { Metronidazole } & \square \text { Glycopeptides } \\ \square \text { Others } & \end{array}$

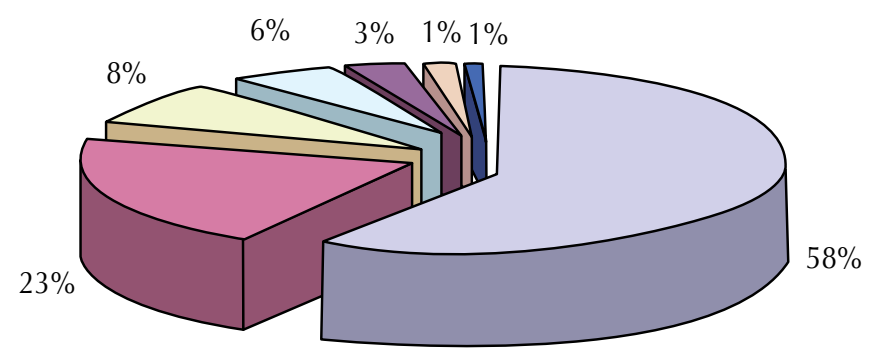

\section{Figure 1: Prescription rate of different antimicrobial classes}

The most common prescribed classes of antimicrobial agent are Cephalosporins (58\%), Penicillins (23\%), Quinolones (8\%), Aminoglycosides (6\%), Metronidazole (3\%) and Glycopeptides (1\%).

Table I: Infection rate during the study period

\begin{tabular}{lccc}
\multicolumn{1}{c}{ Infection } & \multicolumn{2}{c}{ Infected cases } & \% of Infection rate \\
& No. & \% & $(\mathbf{n = 6 3 9 )}$ \\
\hline Community acquired infection: & 18 & 21.40 & 2.80 \\
SSI & 15 & 17.90 & 2.30 \\
RTI & 3 & 3.50 & 0.50 \\
\hline Nosocomial infection: & 66 & 78.60 & 10.30 \\
SSI & 30 & 35.70 & 4.70 \\
UTI & 18 & 21.40 & 2.80 \\
RTI & 15 & 17.90 & 2.30 \\
BSI & 3 & 3.60 & 0.50 \\
\hline Total & 84 & 100.00 & 13.10 \\
Infection rate $=$ (number of infected cases/total number of patients included in the study) $\times 100$. \\
SSI: surgical site infection, UTI: urinary tract infection, RTI: respiratory tract infection, BSI: blood stream \\
infection
\end{tabular}


Table II: Prescription rate of each prescribed antimicrobial agent

Antimicrobial course

\begin{tabular}{|c|c|c|c|c|c|c|c|c|}
\hline \multirow{2}{*}{$\begin{array}{c}\text { Antimicrobial } \\
\text { agent }\end{array}$} & \multicolumn{2}{|c|}{ Prophylactic } & \multicolumn{2}{|c|}{ Random } & \multicolumn{2}{|c|}{ Empiric/therapeutic } & \multicolumn{2}{|c|}{$(n=1119)$} \\
\hline & No & $\%$ & No & $\%$ & No & $\%$ & No & $\%$ \\
\hline Cefotaxime & 342 & 72.60 & 69 & 14.60 & 60 & 12.70 & 471 & 42.10 \\
\hline $\begin{array}{l}\text { Amoxicillin- } \\
\text { flucloxacillin }\end{array}$ & 105 & 77.80 & 15 & 11.10 & 15 & 11.10 & 135 & 12.10 \\
\hline $\begin{array}{l}\text { Ampicillin- } \\
\text { sulbactam }\end{array}$ & 66 & 73.40 & 3 & 3.30 & 21 & 23.30 & 90 & 8.00 \\
\hline Ciprofloxacin & 42 & 46.70 & 30 & 33.30 & 18 & 20.00 & 90 & 8.00 \\
\hline Cefipime & 42 & 73.70 & 6 & 10.50 & 9 & 15.80 & 57 & 5.10 \\
\hline Cefradine & 45 & 100.00 & 0 & 0.00 & 0 & 0.00 & 45 & 4.00 \\
\hline Amikacin & 6 & 14.30 & 0 & 0.00 & 36 & 85.70 & 42 & 3.80 \\
\hline Others & 99 & 52.40 & 42 & 22.20 & 48 & 25.40 & 189 & 16.90 \\
\hline
\end{tabular}

Prescription rate $=$ (the number of antimicrobial agents prescribed in each category /the total number of antibiotics prescribed) $\times 100$

while the appropriateness of empiric $(21 ; 18.9 \%)$ and therapeutic $(69 ; 71.9 \%)$ antibiotic therapies were based mainly on the source of infection $(34 ; 30.6 \%$ and 73 ; $76 \%$, respectively), age of the patient $(59 ; 53.2 \%$ and 89; 92.7\%, respectively), underlying kidney diseases (74; $66.7 \%$ and $81 ; 84.4 \%$, respectively) and site of infection (Table III).

In order to address the relationship between misuse of antibiotics and the development of resistant bacterial strains, all infected cases were subjected to standard microbiological examination of different isolates with analysis of their antibiograms. Ninety-three isolates were recovered from 84 infected patients. The majority of isolates were gram negative ( 77.4 $\%$ ) which are represented by 5 bacterial types (Table IV). The rate of oxacillin resistance was nearly similar among both $S$. aureus and CNS isolates, at $53.3 \%$ and $66.7 \%$, respectively. All isolates of staphylococci were susceptible to vancomycin (Table V). Resistance rates of $\geq 50 \%$ to ceftazidime and/or cefotaxime were detected especially in Klebsiella spp, Pseudomonas spp and E. coli and Acinetobacter strains. Noticeably, susceptibility to imipenem for gram negative bacilli was $>80 \%$ (Table VI).

Methicillin resistance was detected in $57.1 \%$ of Staphylococcal isolates with MRSA prevalence of $38.1 \%(8 / 21$ isolates). Multi-resistant strains represented $48.6 \%(35 / 72)$ of the isolated gram negative strains. ESBLs were identified in $21 / 72 ; 29.2 \%$ of those isolates with percentages of $62.5 \%$ and $33.3 \%$ in Klebsiella spp and $E$. coli isolates, respectively. Of notice, $57.1 \%$ and $66.7 \%$ of Pseudomonas spp and Acinetobacter spp isolates (19.4\% of gram negative isolates) were resistant to amikacin, ciprofloxacin and ceftazidime (Table VII).

\section{Discussion}

Much concern has been voiced in the last two decades about the widespread use of antimicrobial agents, including broad-spectrum antibiotics, leading 
Table III: Appropriateness of empiric and therapeutic antimicrobial therapy in different sources and types of infection

\begin{tabular}{lcccc} 
& Total empiric & Appropriate & $\begin{array}{c}\text { Total } \\
\text { therapeutic }\end{array}$ & Appropriate \\
& $\mathbf{n}$ & $\mathbf{\%}$ & $\mathbf{n}$ & $\mathbf{\%}$ \\
\hline Community acquired Infection (18): & 27 & 22.20 & 21 & 90.50 \\
SSI (15) & 22 & 22.70 & 15 & 86.70 \\
RTI(3) & 5 & 20.00 & 8 & 75.00 \\
\hline Nosocomial infection (66): & 84 & 17.90 & 75 & 66.70 \\
\hline SSI (30) & 36 & 16.70 & 33 & 78.80 \\
UTI(18) & 24 & 25.00 & 21 & 66.70 \\
RTI(15) & 18 & 16.70 & 15 & 40.00 \\
BSI(3) & 6 & 0.00 & 6 & 66.70 \\
\hline Total & 111 & 18.90 & 96 & 71.90 \\
\hline
\end{tabular}

The appropriateness of therapy used $=$ (the number of appropriate antimicrobial agents prescribed $/$ the total number of antibiotics prescribed) $\times 100$.

SSI: surgical site infection, UTI: urinary tract infection, RTI: respiratory tract infection, BSI: blood stream infection

Table IV: The frequency of different isolated organisms

\begin{tabular}{|c|c|c|c|c|c|c|}
\hline \multirow{2}{*}{ Organism } & \multirow{2}{*}{$\begin{array}{l}\text { Total } \\
\text { n (\%) }\end{array}$} & \multirow{2}{*}{$\begin{array}{c}\text { \%Community acquired } \\
\text { infection }\end{array}$} & \multicolumn{4}{|c|}{$\%$ Nosocomial Infections } \\
\hline & & & SSI & RTI & UTI & BSI \\
\hline Staphylococci: & $21(22.60)$ & 28.60 & 42.80 & 14.30 & 0.00 & 14.30 \\
\hline S. aureus & $15(71.40)$ & 40.00 & 40.00 & 20.00 & - & - \\
\hline CNS & $6(28.60)$ & - & 50.00 & - & - & 50.00 \\
\hline Gram -ve: & $72(77.40)$ & 25.00 & 29.20 & 16.70 & 29.20 & - \\
\hline Klebsiella spp & $24(33.30)$ & 37.50 & 12.50 & 25.00 & 25.00 & - \\
\hline Pseudomonas spp & $21(29.20)$ & 28.60 & 42.90 & 14.30 & 14.30 & - \\
\hline E. coli & $18(25.00)$ & - & 33.30 & 16.70 & 50.00 & - \\
\hline Proteus spp & $6(8.30)$ & 50.00 & 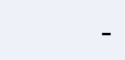 & - & 50.00 & - \\
\hline Acinetobacter spp & $3(4.20)$ & - & 100.00 & - & - & - \\
\hline
\end{tabular}

CNS: coagulase negative staphylococci, S. aureus: Staphylococcus aureus, E. coli: Escherichia coli. SSI: surgical site infection, UTI: urinary tract infection, RTI: respiratory tract infection, BSI: blood stream infection 
Table V: Percentage resistance of gram positive isolates

\begin{tabular}{|c|c|c|c|c|c|c|c|c|c|c|}
\hline Staphylococci & 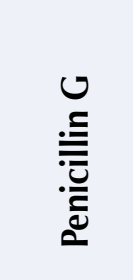 & 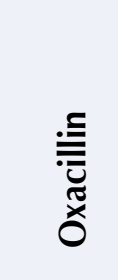 & 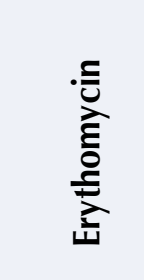 & 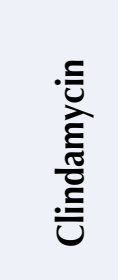 & : & & 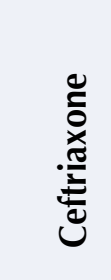 & 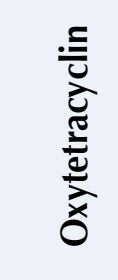 & 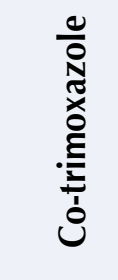 & 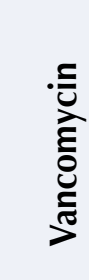 \\
\hline & \multicolumn{10}{|c|}{$\%$ resistance } \\
\hline gureus $(n=15)$ & 86.70 & 53.30 & 100.00 & 86.70 & 60.00 & 80.00 & 33.30 & 73.30 & 86.70 & 0.00 \\
\hline VS $(n=6)$ & 100.00 & 66.70 & 83.30 & 66.70 & 66.70 & 83.30 & 50.00 & 66.70 & 83.30 & 0.00 \\
\hline tal $(n=21)$ & 90.50 & 57.10 & 95.20 & 81.00 & 61.90 & 81.00 & 38.10 & 71.40 & 85.70 & 0.00 \\
\hline
\end{tabular}

CNS: coagulase negative staphylococci.

S. aureus : Staphylococcus aureus

Table VI: Percentage resistance of gram negative isolates

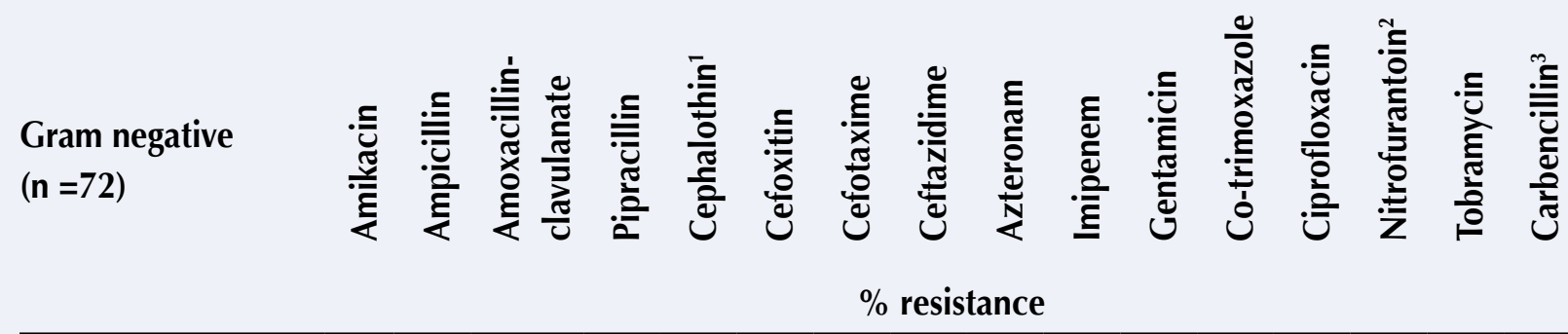

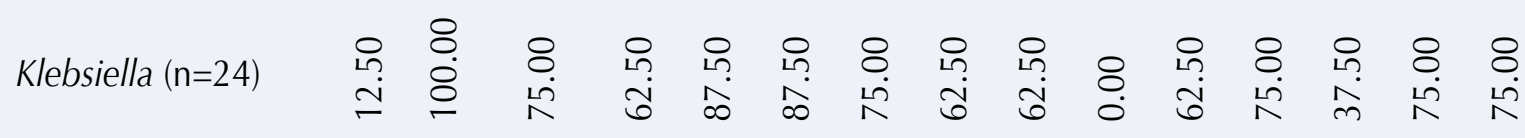

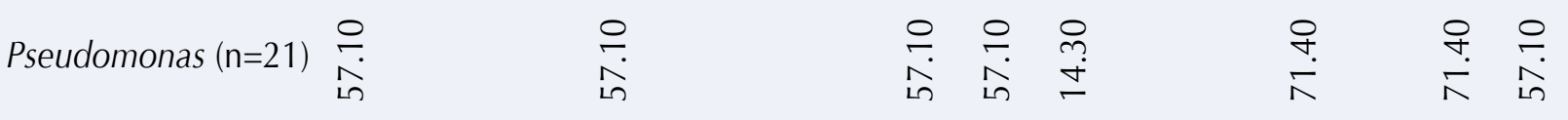

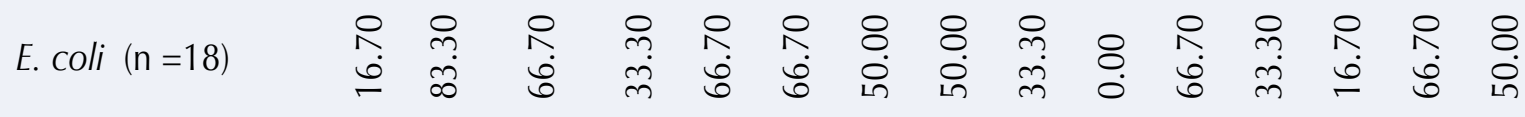

\begin{tabular}{|c|c|c|c|c|c|c|c|c|c|c|c|}
\hline Proteus $(\mathrm{n}=6)$ & 8 & $\begin{array}{l}8 \\
\text { ¿ } \\
\text { 요 }\end{array}$ & $\begin{array}{l}8 \\
\text { ¿ } \\
\text { டी }\end{array}$ & $\begin{array}{l}8 \\
\text { ¿ } \\
\text { டी }\end{array}$ & 8 & \& & $\begin{array}{l}8 \\
\dot{0} \\
\text { in }\end{array}$ & $\begin{array}{l}8 \\
\dot{8} \\
8\end{array}$ & $\stackrel{8}{8}$ & $\begin{array}{l}8 \\
0 \\
0\end{array}$ & $\begin{array}{l}8 \\
0\end{array}$ \\
\hline
\end{tabular}

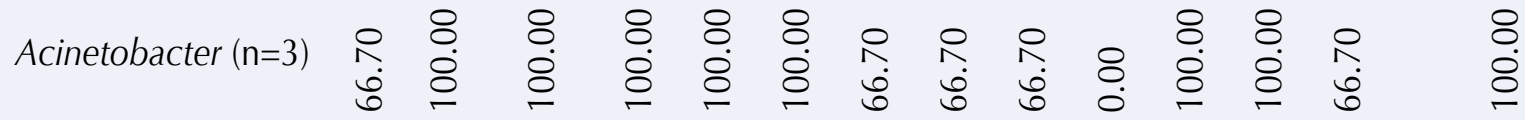

${ }^{1}$ Cephalothin or Cefazolin, ${ }^{2}$ For urinary isolates only, ${ }^{3}$ For Pseudomonas isolates only

E. coli: Escherichia coli 
Table VII: Incidence of antimicrobial multi-resistance in different isolates

\begin{tabular}{|c|c|c|}
\hline Type of resistant organism & $\begin{array}{c}\text { Total number of organism } \\
\text { isolated }\end{array}$ & $\%$ of resistant strains \\
\hline MRSA $^{1}$ & 21 & 57.10 \\
\hline $\mathrm{ESBL}^{2}$ & 72 & 29.20 \\
\hline Klebsiella spp & 24 & 62.50 \\
\hline E. coli & 18 & 33.30 \\
\hline Multi-resistance* & 14 & 19.40 \\
\hline Pseudomonas spp & 21 & 57.10 \\
\hline Acinetobacter & 3 & 66.70 \\
\hline
\end{tabular}

${ }^{1}$ Methicillin Resistant Staphylococcus aureus

${ }^{2}$ Total gram -ve isolates includes 6 Proteus isolates

to emergence of multiple-drug-resistant organisms. In addition, it has been repeatedly demonstrated that $40-70 \%$ of use of these agents is inappropriate, with consequent waste of resources. Many hospitals have attempted to modify these worrying trends by supervising use of certain antimicrobial agents, with various degrees of success. Drug use evaluations have been shown to complement these efforts, and may provide such valuable information. ${ }^{12}$

The purpose of the current study is to generate up-to-date information on antimicrobial use rate and appropriateness, the prevalence of multiresistant bacterial strains and their correlation in our Orthopaedic Department in order to spot light on the situation in our hospital and to establish an effective antibiotic policy.

Almost all the surveyed hospitalized patients in this work have received antibiotics mainly as ABP. Little is known about the antibiotic usage in orthopaedic departments. However, a European study from the early 1990s in the five largest European countries reported that antibiotic usage ranged from $42 \%-55 \%$ with the largest usage of antibiotics was for orthopaedic surgical procedures where antibiotic usage as prophylaxis by the surgical specialties consistent $75 \%$ of therapy days. ${ }^{13}$ Similar Results were obtained by a prospective survey of 3 months in 10 acute care hospitals in Lebanon. ${ }^{14}$ In contrary, Raveh et al., ${ }^{12}$ using a prospective longitudinal method to assess their hospital, found that $62 \%$ of admitted patients and $19 \%$ of orthopaedic patients had received an antimicrobial on evaluation. They explain this difference by the presence of case mix variation as well as the use of different methodology. Moreover, we believe that our high percentage reflects that there is not only misuse but also overuse of antimicrobial agents as $22 \%$ of our patients received random antibiotics.

About $15 \%$ of the prescribed antibiotics were given for infections acquired in the hospital. The most frequent are infections of surgical wounds. Consistent with our results, European studies suggest a healthcare asscoiated infection rate in the range of $9-12 \%$ in countries such as England, Belgium and Denmark as well as Greek and Tunisian studies. ${ }^{13,15,16}$ However, this figure is considerably higher than that reported from other countries and from an Egyptian study involving the surgical wards and 7 ICUs in two hospitals $(0.34 \%)^{17}$ and lower than that reported by Raveh et al., (34\%). ${ }^{12}$ Differences in case mix between hospitals, difference in definition of healthcare 
associated infections and/or failure of hospital hygiene may explain these variations.

Low rate of appropriateness of antimicrobial drug usage was observed in this work. In $\mathrm{ABP}$, both the type and the duration of the chosen antimicrobial agents were responsible for such inappropriateness. Although, the corresponding of antimicrobial agent to the age and kidney conditions had some influence on the failure of antimicrobial therapy, the main factor for the inappropriateness in empirical antimicrobial therapy was the unsuitability of the antimicrobial agent to the site of infection while the main factor for the inappropriateness in therapeutic antimicrobials was inappropriateness of the used antibiotic sensitivity test. Of notice, more than one third of infected cases received repeated empirical courses with the same or different antimicrobial agents and in about $1 / 4$ of those cases the antibiotic sensitivity test was not requested before the start of antibiotic therapy. Overlap in antibiotic courses was obvious.

Various studies published in the last three decades indicate that, using disparate criteria and methods, $40-70 \%$ of antibiotic use is inappropriate. ${ }^{18}$ Consistent with our results, El-Kholy et al., ${ }^{19}$ found that $>80 \%$ of hospitalized patients were given antibiotics on no sound ground within whom $>30 \%$ had received repeated courses, with no apparent reasons for doing so. The reason of these dissimilar rates may be related to whether or not an antibiotic policy is applied. However, we are not aware of any studies that compare rates of appropriate antibiotic use between similar departments, one with well-organized protocols and one without.

The striking finding in this work is the high degree of antimicrobial resistance among the isolates studied. Resistance among Gram-positive cocci and Gramnegative bacilli was widespread in our department. We do not have epidemiological or clinical data to evaluate further the extent to which these resistance patterns reflect endemic antimicrobial resistance within the community, versus hospital spread of resistant organisms within and between various hospitals.
Staphylococcal isolates were highly resistant to all antimicrobials tested, except vancomycin. Our resistant rates were not only similar to those reported in other Egyptian studies but also those reported from other geographical areas. ${ }^{16,17,19}$ On the other hand, resistance rates in the USA and Canada were $26.2 \%$ and $2.7 \%$, respectively. Compared with our isolates, the Canadian isolates of $S$. aureus were also more susceptible to gentamicin, macrolides and co-trimoxazole. ${ }^{20}$

About two thirds of Gram negative bacilli were the two virulent organisms Klebsiella spp and Pseudomonas spp. Antimicrobial resistance among Gram-negative bacilli was common in the present study comparable to reports from other parts of the world. ${ }^{21}$ Susceptibility of Klebsiella spp, Proteus spp and E. coli to ampicillin, ampicillin-sulbactam, and to co-trimoxazole were low. On the other hand, imipenem, aminoglcosides and ciprofloxacin retained activity against most of these isolates.

About half of Pseudomonas and Acinetobacter isolates were resistant to aminoglycosides, ceftazidime, fluoroquinolones and azteronam while, more than $1 / 3$ of Klebsiella spp and E. coli isolates were resistant to ceftazidime, cefotaxime and/or aztreonam. Of notice, the resistance pattern detected in Klebsiella spp and E. coli is a marker for the presence of ESBL. ${ }^{10}$ We did not perform confirmation tests or genetic analyses to confirm the presence of ESBL enzymes in these isolates but the combined resistance pattern suggests that ESBL enzymes are endemic in our department. Of notice, the prevalence of ESBL enzymes has been increasing in many parts of the world. ${ }^{22}$

In summary, our data suggest that antimicrobial resistance among Gram-positive cocci and Gramnegative bacilli is common and significant in orthopaedic department. An inappropriate scheme of antibiotic usage present in our hospitals may be responsible. Particularly alarming are the high rates of ESBL enzymes. Our results call for further epidemiological studies to define whether ESBL are highly endemic in the community and, on a larger scale, for the implementation of a regional and nationwide surveillance system to monitor antimicrobial resistance trends in our hospital and in Egypt. Till this is complete, prescription of antibiotics should be limited. 


\section{Previous presentation}

This paper was accepted and presented in part at the 137th American Public Health Association Annual Meeting, Philadelphia, Pennsylvania, USA, November 2009.

\section{References}

1. Meyer E, Schwab F, Gastmeier P, Rueden H, Daschner FD. Surveillance of antimicrobial use and antimicrobial resistance in German intensive care units (SARI): a summary of the data from 2001 through 2004. Infection 2006; 34: 303-309.

2. Monroe S, Polk R. Antimicrobial use and bacterial resistance. Curr Opin Microbiol 2000; 3: 496-501.

3. Frank U, Kleissle EM, Daschner FD, et al. Multicentre study of antimicrobial resistance and antibiotic consumption among 6,780 patients with bloodstream infections. Eur J Clin Microbiol Infect Dis 2006; 25: 815-817.

4. Doyne EO, Alfaro MP, Siegel RM, et al. A randomized controlled trial to change antibiotic prescribing patterns in a community. Arch Pediatr Adolesc Med 2004; 158: 577 583.

5. Duewelhenke N, KrutO, EyselP. Influence on mitochondria and cytotoxicity of different antibiotics administered in high concentrations on primary human osteoblasts and cell lines. Antimicrob Agents Chemother 2007; 51: 54-63.

6. Essack SY, Connolly C, Sturm AW. Antibiotic use and resistance in public-sector hospitals in KwaZulu-Natal. SAMJ 2005; 95: 865-870.

7. Nosomed guidline. Protocol incidence survey of surgical site infection. Feb; 2005.

8. Collee JG, Fraser AG, Marmion BP, Simmons A. Mackie and McCartney practical medical microbiology, $14^{\text {th }}$ ed. New York: Churchill Livingstone; 1996.

9. Clinical and Laboratory Standards Institute (CLIS). Performance standards for antimicrobial susceptibility testing; Nineteenth informational supplement. CLIS document M100-S19.

10. New and updated protocols for antimicrobial susceptibility testing of pathogens under EARSS surveillance; 2005. http:// www.earss.rivm.nl

11. Abou YZ, Alwan AAS. WHO regional publications, Eastern Mediterranean Series. Guide to chemotherapy and chemoprophylaxis in bacterial infections. Alexandria: Bafra Graphics; 1998.

12. Raveh D, Levy Y, Schlesinger Y, Greenberg A, Rudensky B, Yinnon AM. Longitudinal surveillance of antibiotic use in the hospital. Q J Med 2001; 94: 141-152.
13. Halls GA. The management of infections and antibiotics therapy: a European survey. I Antimicrob Chemother 1993; 31: 985-1000.

14. Azzam RA, Hamade NN. Major reasons to prescribe antimicrobial prophylaxis in surgery. Int Surg 2003; 88:105108.

15. Gikas A, Pediaditis I, Roumbelaki M, Troulakis G, Romanos J, Tselentis Y. Repeated multi-center prevalence surveys of hospital-acquired infection in Greek hospitals. J Hosp Infect 1999; 41: 11-18.

16. Kallel H, Bahoul M, Ksibi $H$, et al. Prevalence of hospitalacquired infection in a Tunisian hospital. J Hosp Infect 2005; 59: 343-347.

17. Saied GM. Microbial pattern and antimicrobial resistance, a surgeon's perspective: retrospective study in surgical wards and seven intensive-care units in two University Hospitals in Cairo, Egypt. Dermatology 2006; 212(suppl 1): 8-14.

18. Drori-Zeides T, Raveh D, Schlesinger Y, Yinnon AM. Practical guidelines for vancomycin usage, with prospective drug utilization evaluation. Infect Control Hosp Epidemiol 2000; 21: 45-48.

19. El Kholy A, Baseem H, Hall GS, Procop GW, Longworth DL. Antimicrobial resistance in Cairo, Egypt 1999-2000: a survey of five hospitals. J Antimicrob Chemother 2003; 51: 625-630.

20. Pfaller MA, Jones RN, Doern GV, Kugler K. Bacterial pathogens isolated from patients with bloodstream infection: frequencies of occurrence and antimicrobial susceptibility patterns from the SENTRY Antimicrobial Surveillance Program (United States and Canada) 1997. Antimicrob Agents Chemother 1998; 42: 1762-1770.

21. Oplustil CP, Nunes R, Mendes C. Multicenter evaluation of resistance patterns of Klebsiella pneumoniae, Escherichia coli, Salmonella spp., and Shigella spp. isolated from clinical specimens in Brazil: RESISTNET Surveillance Program. Brazil J Infect Dis 2001; 5: 8-12.

22. Winokur PL, Canton R, Casellas JM, Legakis N. Variations in the prevalence of strains expressing an extended spectrum B-lactamase phenotype and characterization of isolates from Europe, the Americas, and the Western Pacific region. Clin Infect Dis 2001; 32: S94-103. 